\title{
Temperature and Stress Distribution in a Non-simple Elastic Elliptical Plate due to Point Impulsive Heat Sources
}

\author{
Pravin Bhad ${ }^{1}$, Vinod Varghese ${ }^{2}$, Ashwini Mahakalkar ${ }^{3}$ \\ ${ }^{1}$ Department of Mathematics, Priyadarshini J. L. College of Eng., Nagpur, India \\ ${ }^{2}$ Department of Mathematics, Smt. Sushilabai Bharti Science College, Arni, Yavatmal, India \\ ${ }^{3}$ Department of Mathematics, Mahatma Gandhi Science College, Armori, Gadchiroli, India \\ E-mail: ${ }^{2}$ vino7997@gmail.com
}

Received 16 January 2019, Revised 05 March 2019, Revised 27 March 2019, Accepted 27 March 2019

\begin{abstract}
The paper attempts to determine the thermoelastic stresses in a thin elliptical plate made up of non-simple elastic material subjected to point impulsive time-dependent source of heat moving with constant velocity over the specified finite portion. The temperature field in the plate has been considered when the sectional heat supply is continuously distributed along the circumference of an ellipse over the upper face with zero temperature on the lower face, and thermally insulated curved edge. The solution is formulated involving the Mathieu and modified functions by employing the Laplace transform technique. The analytical solution for the thermal stress components is obtained using Airy's stress function with mechanical boundary conditions as stress-free. Numerical results are also obtained.
\end{abstract}

\section{Keywords: Thermal load; elliptical plate; heat conduction; stresses; Mathieu functions.}

\section{Introduction}

The theoretical study of the heat flow within a thin elliptical Plate is of considerable practical importance in a wide range of fields such as mechanical, aerospace and food engineering fields throughout the past years. The twotemperature model is one of the non-classical thermoelasticity theories of elastic solids. The classification of real materials into simple and non-simple materials was proposed by Chen and Gurtin [1] for which thermodynamic and conductive temperature were not identical for nonsimple materials, unlike simple materials. The studies were further extended to deformable bodies by Chen et al. [2] and shown that such materials contain an additional term involving the time derivative of the Laplacian of the conductive temperature. Considering isotropy and linearity, for such materials, they have shown that the two temperatures are related by $\phi-T=b \nabla^{2} T, b \geq 0$, in which $\phi$ is the thermodynamic temperature, $T$ is the conductive temperature and $b$ is the temperature discrepancy factor. The key element that sets the two-temperature thermoelasticity theory apart from the classical theory is the material parameter. Specifically, in the limit as $b \rightarrow 0$, $\varphi \rightarrow T$ and the classical theory is recovered. Problems of determination of thermal stresses in the simple material under various mechanical and thermal boundary conditions have been considered earlier by many researchers viz. Roy Choudhary [3], Khobragade and Deshmukh [4], Varghese and Khalsa [5] and many others. Similarly, the present author [6-13] have investigated various thermoelastic problems in elliptical objects of simple materials due to interior heat generation or sectional heat supply with different solid objects. Even one-dimensional thermoelastic problems made up of non-simple elastic material due to heat sources has been studied by various authors, viz. Chakrabarty [14], Laha [15], Dhar [16-17] etc. In recent years, we have also witnessed a number of papers on nonsimple elastic materials vide, Ciarletta [18], Chakraborty [19], Quintanilla [20-22], Zenkour [23] etc. But none of the previous investigators solved the higher dimensional thermoelastic problem for a non-simple material, particularly elliptical objects with the elliptical coordinate system. In the present manuscript, the author has made an attempt to solve the thermoelastic problem in a thin elliptical plate made up of non-simple elastic material. Laplace transform technique has been used to solve the problem. Due to the complicated nature of the integrands of Bromwich integrals, the exact solution of the problem cannot be obtained so easily. Finally, by considering a circle as a special kind of ellipse, it is shown that the temperature distribution and history in an infinite circular solution can be derived as a special case from the present mathematical and illustrated numerically.

\section{Formulation of the Problem}

We consider a thin elliptical elastic plate made up of the non-simple material of radius $\xi_{0}$ and thickness $\ell$ in the space $D=\left\{(\xi, \eta, z) \in R^{3}: \quad 0<\xi<\xi_{0}, \quad 0<\eta<2 \pi\right.$, $-\ell / 2<z<\ell / 2\}$ defined by the transformation $\xi+i \eta=\cosh ^{-1}[(x+i y) / c]$. The geometry of the plate indicates that an elliptical-cylindrical coordinate system $(\xi, \eta, z)$ is the most appropriate choices of the reference frame, which are related to the rectangular coordinate system $(x, y, z)$ by the relation $x=c \cosh \xi \cos \eta$ $y=c \sinh \xi \sin \eta$ and $z=z$. The curves $\eta=\mathrm{constant}$ represent a family of confocal hyperbolas while the curves $\xi=$ constant represent a family of confocal ellipses. The length $2 c$ is the distance between their common foci (refer to Figure 1). Both sets of curves intersect each other 
orthogonally at every point in space. The parameter $\xi$ varies from 0 where it defines the interfocal line to $\xi_{0}$, the coordinate $\eta$ is an angular coordinate taking the range $\eta \in[0,2 \pi)$, and $z \in(0, \ell)$. It is noted that $c$ is denoted as $2 c=\left(a_{0}^{2}-b_{0}^{2}\right)^{1 / 2}$ and $\xi_{0}=\tanh ^{-1}\left(b_{0} / a_{0}\right)$ in terms of the semi-major length $a_{0}$ and the semi-minor length $b_{0}$.

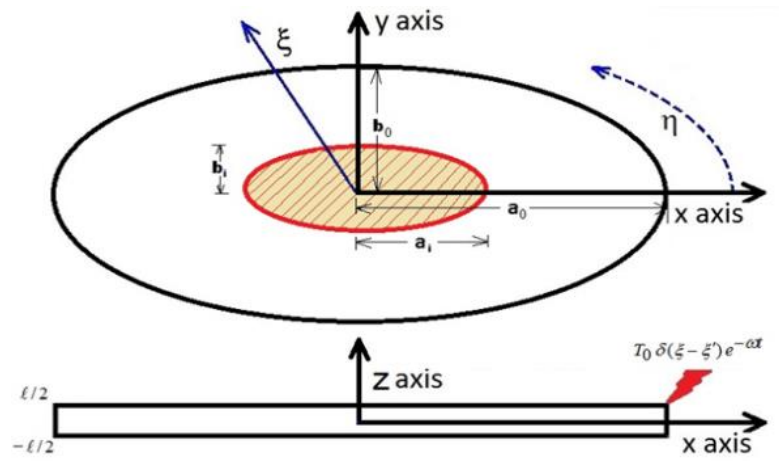

Figure 1. Elliptical plate configuration

\subsection{Heat Conduction Formulation}

The modified heat conduction differential equation for the non-simple material is given as

$$
\begin{gathered}
\left(1+\frac{b}{\kappa} \frac{\partial}{\partial t}\right)\left[h^{2}\left(\frac{\partial^{2} T}{\partial \xi^{2}}+\frac{\partial^{2} T}{\partial \eta^{2}}\right)+\frac{\partial^{2} T}{\partial z^{2}}\right]=\frac{1}{\kappa} \frac{\partial T}{\partial t} \\
-q_{0} \delta(t) \delta(z) \delta\left(\xi-\xi^{\prime}\right) ; 0<\xi^{\prime}<\xi_{i}<\xi_{0}
\end{gathered}
$$

where thermal diffusivity is taken as $\kappa=\lambda / \rho C_{v}, \lambda$ is the thermal conductivity of the material, $\rho$ is the density and $C_{v}$ is the calorific capacity, $T=T(\xi, \eta, t)$ is the temperature distribution, $Q=q_{0} \delta(t) \delta(z) \delta\left(\xi-\xi^{\prime}\right)$ is the internal source function, $\xi_{i}=\tanh ^{-1}\left(b_{i} / a_{i}\right)$, $\xi^{\prime}=\tanh ^{-1}\left(b^{\prime} / a^{\prime}\right)$, and the metric coefficient $h$ is given by $h^{2}=2 /\left[c^{2}(\cosh 2 \xi-\cos 2 \eta)\right]$.

The fundamental Eq. (2) is to be solved subject to the following conditions

$$
\begin{aligned}
& \left.T\right|_{t=0}=0,\left.\frac{\partial T}{\partial \xi}\right|_{\xi=\xi_{0}}=0, \\
& \left.T\right|_{z=-\frac{\ell}{2}}=0,\left.T\right|_{z=\frac{1}{2}}= \begin{cases}f(\xi, \eta) e^{-\omega t} \text { for } 0 \leq \xi \leq \xi_{i} \\
0 & \text { for } \xi_{i} \leq \xi \leq \xi_{0}\end{cases}
\end{aligned}
$$

where $\omega$ is constant, $f(\xi, \eta)=\delta\left(\xi-\xi^{\prime}\right)$ is the prescribed sectional heat supply and $\delta()$ is the Dirac delta function.

\subsection{Thermoelastic Displacement and Stresses}

Since we have assumed that the plate is sufficiently thin, we can assume that the plane, initially normal to the middle or neutral plane $(z=0)$ before bending, remains straight and normal to the middle surface during the deformation. The length of such elements is not altered. This means that the axial stress is negligible compared to the other stress components. This can be neglected in the stress-strain relations. According to the assumption as mentioned earlier, the modified displacements [24] are given by

$u_{\xi}=\frac{h}{2 \mu}\left\{-\frac{\partial}{\partial \xi} \chi(\xi, \eta, t)+\frac{1}{h^{2}} \frac{\partial}{\partial \eta} P(\xi, \eta, t)\right\}$,

$u_{\eta}=\frac{h}{2 \mu}\left\{-\frac{\partial}{\partial \eta} \chi(\xi, \eta, t)+\frac{1}{h^{2}} \frac{\partial}{\partial \xi} P(\xi, \eta, t)\right\}$

in which $\left(u_{\xi}, u_{\eta}\right)$ are displacements in the directions normal to the curves $(\xi, \eta), \chi(\xi, \eta, t)$ is Airy's stress function and $P(\xi, \eta, t)$ is the harmonic function, satisfies the equations as [24]

$\nabla^{2} P=0$,

$\frac{\partial}{\partial \xi}\left(\frac{1}{h^{2}} \frac{\partial P}{\partial \eta}\right)+\frac{\partial}{\partial \eta}\left(\frac{1}{h^{2}} \frac{\partial P}{\partial \xi}\right)=\frac{\lambda+2 \mu}{\lambda+\mu}\left(\frac{\partial^{2} \chi}{\partial \xi^{2}}+\frac{\partial^{2} \chi}{\partial \eta^{2}}\right)$

and the modified stress function in Eq. (4) satisfies below Eq. (5) of the fourth order

$$
h^{2} \nabla^{2} h^{2} \nabla^{2} \chi=-h^{2}\left(1+\frac{b}{\kappa} \frac{\partial}{\partial t}\right) \nabla^{2} T
$$

The components of the stresses [25] are represented as

$$
\begin{aligned}
& \sigma_{\xi \xi}=h^{2} \frac{\partial^{2} \chi}{\partial \eta^{2}}+\frac{c^{2} h^{4}}{2} \sinh 2 \xi \frac{\partial \chi}{\partial \xi}-\frac{c^{2} h^{4}}{2} \sin 2 \eta \frac{\partial \chi}{\partial \eta}, \\
& \sigma_{\eta \eta}=h^{2} \frac{\partial^{2} \chi}{\partial^{2}}-\frac{c^{2} h^{4}}{2} \sinh 2 \xi \frac{\partial \chi}{\partial \xi}+\frac{c^{2} h^{4}}{2} \sin 2 \eta \frac{\partial \chi}{\partial \eta}, \\
& \sigma_{\xi \eta}=-h^{2} \frac{\partial^{2} \chi}{\partial \xi \partial \eta}+\frac{c^{2} h^{4}}{2} \sinh 2 \eta \frac{\partial \chi}{\partial \xi}+\frac{c^{2} h^{4}}{2} \sinh 2 \xi \frac{\partial \chi}{\partial \eta}
\end{aligned}
$$

For traction free surface the stress functions

$$
\sigma_{\xi \xi}=\sigma_{\xi \eta}=0 \text { at } \xi=\xi_{0}
$$

Thus, the equations (1) to (7) constitute the mathematical formulation under consideration.

\section{Solution to the Problem}

\subsection{Solution for the Temperature Distribution} obtains

Now applying Laplace transform to Eq. (2), one

$\left(1+\frac{b}{\kappa} p\right)\left[h^{2}\left(\frac{\partial^{2}}{\partial \xi^{2}}+\frac{\partial^{2}}{\partial \eta^{2}}\right)+\frac{\partial^{2}}{\partial z^{2}}\right] \bar{T}=\frac{p}{\kappa} \bar{T}-q_{0} \delta(z) \delta\left(\xi-\xi^{\prime}\right)(8)$

with

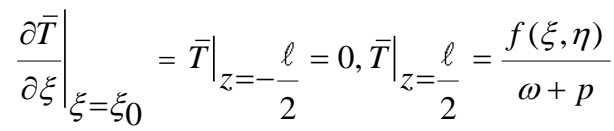

in which $\bar{T}=\bar{T}(\xi, \eta, p)$ is the temperature distribution in a transformed domain.

Now, without loss of generality, we assume the solution of Eq. (8) satisfying the first two conditions given in Eq. (9), as

$$
\begin{aligned}
\bar{T}= & \sum_{n=0}^{\infty} \sum_{m=1}^{\infty} A_{n, m} C_{e_{2 n}}\left(\xi, q_{n, m}\right) c_{e_{2 n}}\left(\eta, q_{n, m}\right) \\
& \times \sinh \left[\alpha_{2 n, m}\left(z+\frac{\ell}{2}\right)\right]
\end{aligned}
$$

where, $\alpha_{n, m}=\left(\gamma_{2 n, m}^{2}+p / \kappa\right)^{1 / 2}, A_{n, m}$ is a constant that has to be determined from the nature of temperature on the 
upper face, $\gamma_{2 n, m}=-4 q_{n, m} / c^{2}, q_{n, m}$ is the root of the equation $C_{e_{2 n}}\left(\xi_{0}, q\right)=0$ and according to McLachlan [26, pp. 21,27]

$$
\begin{gathered}
C_{e_{2 n}}\left(\xi, q_{n, m}\right)=\sum_{r=0}^{\infty} A_{2 r}^{(2 n)} \cosh 2 r \xi \\
c_{e_{2 n}}\left(\eta, q_{n, m}\right)=\sum_{r=0}^{\infty} A_{2 r}^{(2 n)} \cos 2 r \eta
\end{gathered}
$$

in which $A$ 's being the functions of $q$; then at any point within the range [26, pp. 296]

$$
f(\xi, \eta)=\sum_{n=0}^{\infty} \sum_{m=1}^{\infty} B_{n, m} C_{e_{2 n}}\left(\xi, q_{n, m}\right) c_{e_{2 n}}\left(\eta, q_{n, m}\right)
$$

Hence, by the theory of the Mathieu Function [26], one obtains

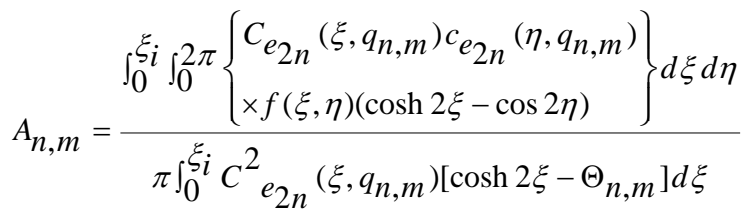

where

$$
\pi \Theta_{n, m}=\int_{0}^{2 \pi} c^{2} e_{2 n}\left(\eta, q_{n, m}\right) \cos 2 \eta d \eta
$$

Now from Eqs. (9)-(11), one yield

$$
\begin{aligned}
\bar{T}= & \sum_{n=0}^{\infty} \sum_{m=1}^{\infty} A_{n, m} C_{e_{2 n}}\left(\xi, q_{n, m}\right) c_{e_{2 n}}\left(\eta, q_{n, m}\right) \\
& \times\left\{\frac{\sinh \left[\left(\gamma_{2 n, m}^{2}+\frac{p}{\kappa}\right)^{1 / 2}\left(z+\frac{\ell}{2}\right)\right]}{(p+\omega) \sinh \left[\left(\gamma_{2 n, m}^{2}+\frac{p}{\kappa}\right)^{1 / 2} \ell\right]}\right\}
\end{aligned}
$$

Now by inverting Eq. (12) by Laplace's inversion theorem, one obtains the following Bromwich integral as

$$
\begin{aligned}
T= & \frac{1}{2 \pi i} \sum_{n=0}^{\infty} \sum_{m=1}^{\infty}\left\langle A_{n, m} C_{e_{2 n}}\left(\xi, q_{n, m}\right) c_{e_{2 n}}\left(\eta, q_{n, m}\right)\right. \\
& \left.\times \int_{\hat{c}-i \infty}^{\widehat{c}+i \infty}\left\{\frac{\sinh \left[\left(\gamma_{2 n, m}^{2}+\frac{p}{\kappa}\right)^{1 / 2}\left(z+\frac{\ell}{2}\right)\right]}{(p+\omega) \sinh \left[\left(\gamma_{2 n, m}^{2}+\frac{p}{\kappa}\right)^{1 / 2} \ell\right]}\right\} d p\right\rangle
\end{aligned}
$$

where $\hat{c}$ is a real number, such that $\hat{c}+i \infty$ is in the halfplane of convergence.

To evaluate the Bromwich integral in Eq. (13) by contour integration method, we have to calculate the residue at the poles of the integrand

$$
I=\frac{1}{2 \pi i} \int_{\hat{c}-i \infty}^{\hat{c}+i \infty}\left\{\frac{\sinh \left[\left(\gamma_{2 n, m}^{2}+\frac{p}{\kappa}\right)^{1 / 2}\left(z+\frac{\ell}{2}\right)\right]}{(p+\omega) \sinh \left[\left(\gamma_{2 n, m}^{2}+\frac{p}{\kappa}\right)^{1 / 2} \ell\right]}\right\} d p
$$

$$
\begin{aligned}
& \text { Now } \\
& \begin{array}{l}
\sinh \left[\left(\gamma_{2 n, m}^{2}+\frac{p}{\kappa}\right)^{1 / 2} \ell\right]=0 \Rightarrow\left(\gamma_{2 n, m}^{2}+\frac{p}{\kappa}\right)^{1 / 2} \ell=\frac{i \pi v}{\ell} \\
\Rightarrow \gamma_{2 n, m}^{2}+\frac{p}{\kappa}=-\frac{\pi^{2} v^{2}}{\ell^{2}} \\
\Rightarrow p=p_{v}=-\kappa\left(\beta_{v}^{2}+\gamma_{2 n, m}^{2}\right), v=0,1,2 . . \infty
\end{array}
\end{aligned}
$$

in which $\beta_{v}=\pi v / \ell$.

In this case, the poles of the integrand are at the following points

(i) Poles at $p=p_{v}$

$$
\begin{gathered}
\left.\lim _{p \rightarrow p_{v}\left(\frac{p-p_{v}}{p+\omega}\right)}\left\{\frac{\sinh \left[\left(\gamma_{2 n, m}^{2}+\frac{p}{\kappa}\right)^{1 / 2}\left(z+\frac{\ell}{2}\right)\right]}{\sinh \left[\left(\gamma_{2 n, m}^{2}+\frac{p}{\kappa}\right)^{1 / 2} \ell\right]}\right\} e^{p t}\right] \\
=\frac{2 \pi v(-1)^{v+1} \sin \left[\beta_{v}\left(z+\frac{\ell}{2}\right)\right]}{\kappa \ell^{2}\left(\beta_{v}^{2}+\gamma_{2 n, m}^{2}\right)^{2}} e^{-\kappa\left(\beta_{v}^{2}+\gamma_{2 n, m}^{2}\right)}
\end{gathered}
$$

(ii) Residue at pole $p=-\omega$

$$
\begin{aligned}
\lim _{p \rightarrow-\omega}\left\{\frac{\sinh \left[\left(\gamma_{2 n, m}^{2}+\frac{p}{\kappa}\right)^{1 / 2}\left(z+\frac{\ell}{2}\right)\right]}{\sinh \left[\left(\gamma_{2 n, m}^{2}+\frac{p}{\kappa}\right)^{1 / 2} \ell\right]}\right\} e^{-\omega t} \\
\left.\left.=\left\{\frac{\sinh \left[\left(\gamma_{2 n, m}^{2}-\frac{\omega}{\kappa}\right)^{1 / 2}\left(z+\frac{\ell}{2}\right)\right]}{\sinh \left[\left(\gamma_{2 n, m}^{2}-\frac{\omega}{\kappa}\right)^{1 / 2}\right]}\right\} e^{-\omega t}\right]{ }^{-\omega t}\right]
\end{aligned}
$$

Now by the residue theorem, the integrand given in Eq. (14) can be written as

$$
\begin{gathered}
I=\frac{1}{2 \pi i}\left\{\frac{2 \pi v(-1)^{v+1} \sin \left[\beta_{v}\left(z+\frac{\ell}{2}\right)\right]}{\kappa \ell^{2}\left(\beta_{v}^{2}+\gamma_{2 n, m}^{2}\right)} e^{-\kappa\left(\beta_{v}^{2}+\gamma_{2 n, m}^{2}\right)}\right. \\
\left.+\frac{\sinh \left[\left(\gamma_{2 n, m}^{2}-\frac{\omega}{\kappa}\right)^{1 / 2}\left(z+\frac{\ell}{2}\right)\right]}{\sinh \left[\left(\gamma_{2 n, m}^{2}-\frac{\omega}{\kappa}\right)^{1 / 2} \ell\right]} e^{-\omega t}\right\}
\end{gathered}
$$

Thus, the temperature field can be obtained from Eq. (13) using Eq. (15) as 


$$
\begin{aligned}
& T=\frac{1}{2 \pi i} \sum_{n=0}^{\infty} \sum_{m=1}^{\infty} A_{n, m} C_{e_{2 n}}\left(\xi, q_{n, m}\right) c_{e_{2 n}}\left(\eta, q_{n, m}\right) \\
& \times\left\{\begin{array}{l}
\frac{2 \beta_{V}(-1)^{\nu+1}}{\kappa \ell} \frac{\sin \left[\beta_{V}\left(z+\frac{\ell}{2}\right)\right]}{\beta_{V}^{2}+\gamma^{2} 2 n, m} e^{-\kappa\left(\beta_{V}^{2}+\gamma_{2 n, m}^{2}\right)} \\
+\frac{\sinh \left[\left(\gamma_{2 n, m}^{2}-\frac{\omega}{\kappa}\right)^{1 / 2}\left(z+\frac{\ell}{2}\right)\right]}{+\sinh \left[\left(\gamma_{2 n, m}^{2}-\frac{\omega}{\kappa}\right)^{1 / 2} \ell\right]} e^{-\omega t}
\end{array}\right\}
\end{aligned}
$$

\subsection{Solution for displacement and its stresses}

Assuming Airy's stress function $\chi(\xi, \eta, t)$, which satisfies Eq. (5) as

$$
\begin{aligned}
\chi= & \frac{-h^{2} c^{2}}{4 \pi i} \sum_{n=0}^{\infty} \sum_{m=1}^{\infty} \frac{A_{n, m}}{q_{2 n, m}}\left[C_{n, m} \xi+D_{n, m} \eta+\xi^{2} \eta\right] \\
& \times\left\{\begin{array}{l}
\frac{2 \beta_{v}(-1)^{v+1}}{\kappa \ell} \frac{\sin \left[\beta_{v}\left(z+\frac{\ell}{2}\right)\right]}{\beta_{v}^{2}+\gamma_{2 n, m}^{2}} e^{-\kappa\left(\beta_{v}^{2}+\gamma_{2 n, m}^{2}\right)} \\
\left.+\frac{\sinh \left[\left(\gamma_{2 n, m}^{2}-\frac{\omega}{\kappa}\right)^{1 / 2}\left(z+\frac{\ell}{2}\right)\right]}{\sinh \left[\left(\gamma_{2 n, m}^{2}-\frac{\omega}{\kappa}\right)^{1 / 2}\right]} e^{-\omega t}\right]
\end{array}\right\}
\end{aligned}
$$

where $C_{n, m}$ and $D_{n, m}$ are arbitrary functions and can be obtained by using traction free conditions given in Eq. (7) as

$$
\begin{aligned}
C_{n, m}= & \xi_{0}^{2}\left\{c e _ { n } ( \eta , q _ { 2 n , m } ) \left[c ^ { 2 } h ^ { 2 } \eta \operatorname { s i n } 2 \eta \left(-2+c^{2} h^{2}\right.\right.\right. \\
& \left.\left.\times \sin 2 \eta)-c^{4} h^{4} \eta \sinh ^{2} 2 \xi_{0}\right] \times c e_{n}^{\prime \prime}\left(\eta, q_{2 n, m}\right)\right\} \\
& /\left\{c e_{n}\left(\eta, q_{2 n, m}\right) \times\left[-\xi_{0} c^{4} h^{4} \eta \sin ^{2} 2 \eta+c^{2} h^{2}\right.\right. \\
& \left.\times \sinh 2 \xi_{0}\left(2+\xi_{0} c^{2} h^{2} \sinh 2 \xi_{0}\right)\right] \\
& \left.+2 \xi_{0}\left(2+\xi_{0} c^{2} h^{2} \sinh 2 \xi_{0}\right) c e_{n}^{\prime \prime}\left(\eta, q_{2 n, m}\right)\right\}
\end{aligned}
$$

$$
\begin{aligned}
D_{n, m}= & \xi_{0}\left\{-1+\left[4 \left(-c^{2} h^{2} \sinh 2 \xi_{0} c e_{n}\left(\eta, q_{2 n, m}\right)\right.\right.\right. \\
& \left.+\xi_{0}\left(-2+c^{2} h^{2} \eta \sin 2 \eta\right) c e_{n}^{\prime \prime}\left(\eta, q_{2 n, m}\right)\right] \\
& / c^{2} h^{2} c e_{n}\left(\eta, q_{2 n, m}\right)\left[\xi_{0} c^{2} h^{2}(-2+\cos 4 \eta\right. \\
& \left.\left.+\cosh 4 \xi_{0}\right)+4 \sinh 2 \xi_{0}\right]+4 \xi_{0}\left(2+\xi_{0} c^{2} h^{2}\right. \\
& \left.\left.\times \sinh 2 \xi_{0}\right) c e_{n}^{\prime \prime}\left(\eta, q_{2 n, m}\right)\right\}
\end{aligned}
$$

in which the double prime symbol (") denotes double differentiation with respect to the assigned variable. Similarly, if we assume the solution of the first equation of Eq. (4) satisfying the conditions (7) as

$$
\begin{aligned}
P= & \frac{4}{2} \sum_{n=0}^{\infty} \sum_{m=1}^{\infty} q_{2 n, m} B_{n, m} C_{e_{2 n}}\left(\xi, q_{n, m}\right) \\
& \times c_{e_{2 n}}\left(\eta, q_{n, m}\right)\left\{\frac{2 \beta_{v}}{\kappa \ell}(-1)^{v+1} e^{-\kappa\left(\beta_{v}^{2}+\gamma_{2 n, m}^{2}\right)}\right.
\end{aligned}
$$

$$
\left.\times \frac{\sin \left[\beta_{v}\left(z+\frac{\ell}{2}\right)\right]}{\left(\beta_{\mu}^{2}+\gamma_{2 n, m}^{2}\right)}{\sinh \left[\left(\gamma_{2 n, m}^{2}-\frac{\omega}{\kappa}\right)^{1 / 2}\left(z+\frac{\ell}{2}\right)\right]}_{+} \frac{\sinh \left[\left(\gamma_{2 n, m}^{2}-\frac{\omega}{\kappa}\right)^{1 / 2} \ell\right]}{\ell} e^{-\omega t}\right\}
$$

in which $B_{n, m}$ is the constant to be determined using equation (4) considering Eq. (17), as

$$
\begin{aligned}
& B_{n, m}=\frac{c^{2}}{4 \pi i}\left(\frac{\lambda+2 \mu}{\lambda+\mu}\right) \\
& \times \sum_{\substack{m=1 n=0 \\
4 q_{2 n, m}}}^{\infty}\left\{\frac{A_{n, m}}{c_{2 n, m}}\left[C_{n, m} \xi+D_{n, m} \eta+\xi^{2} \eta\right]\right. \\
&+\frac{C_{e_{2 n}}\left(\xi, q_{n, m}\right) c_{e_{2 n}}\left(\eta, q_{n, m}\right) / F(\xi, \eta)}{}
\end{aligned}
$$

with

$$
\begin{aligned}
F(\xi, \eta)= & \sinh 2 \xi C_{e_{2 n}}\left(\xi, q_{n, m}\right) c e_{2 n}^{\prime}\left(\eta, q_{n, m}\right) \\
& +\sin 2 \eta C e_{2 n}^{\prime}\left(\xi, q_{n, m}\right) c e_{2 n}\left(\eta, q_{n, m}\right)
\end{aligned}
$$

Now putting the expression of Airy's stress function $\chi(\xi, \eta, t)$ from Eq. (17) and harmonic function $P(\xi, \eta, t)$ from Eq. (18), one obtains the expression for the required displacements. Similarly using Eq. (17) in Eq. (6), one obtains the expression for thermal stresses. The resulting equations of displacements (i.e. $u_{\xi}$ and $u_{\eta}$ ) and thermal stresses (i.e. $\sigma_{\xi \xi}, \sigma_{\eta \eta}$ and $\sigma_{\xi \eta}$ ) which are also rather lengthy, and consequently are omitted here for the sake of brevity, but considered during graphical discussion described in below section.

\section{Transition to Circular}

When the elliptic plate tends to a circular structure of the radius $\xi_{0}$, the semi-focal $c \rightarrow 0$ and then $\alpha_{m}$ is the roots of the transcendental equation $J_{0}\left(\alpha_{m}\right)=0$ .Also $e \rightarrow 0 \quad[$ as $\xi \rightarrow \infty], \cosh 2 \xi d \xi \rightarrow$ $2 \cosh 2 \xi \sinh 2 \xi d \xi \rightarrow 2 r d r / c^{2}, \sinh \xi \rightarrow \cosh \xi$, $h \cosh \xi \rightarrow r$ as $h \rightarrow 0], \cosh \xi d \xi \rightarrow r d r$, $h \sinh \xi d \xi \rightarrow d r$, Using results from [26] $C e_{0}\left(\xi, q_{0, m}\right) \rightarrow p_{0}^{\prime} J_{0}\left(\alpha_{m} r\right), c e_{0}\left(\eta, q_{0, m}\right) \rightarrow 1 / \sqrt{2}$, $\gamma_{0, m}^{2}=\gamma_{0, m}^{2} / \xi_{0}^{2}=\alpha_{m}^{2} / \xi_{0}^{2}=\gamma_{m}^{2}$

Considering the aforesaid parameters, the temperature distribution in cylindrical coordinate is finally represented by 


$$
\begin{aligned}
T= & \frac{1}{2 \sqrt{2} \pi i} \sum_{m=1}^{\infty} A_{m} p_{0}^{\prime} J_{0}\left(\alpha_{m} r\right)\left\{\frac{2 \beta_{v}(-1)^{v+1}}{\kappa \ell\left(\beta_{v}^{2}+\gamma_{m}^{2}\right)}\right. \\
& \times \sin \left[\beta_{v}\left(z+\frac{\ell}{2}\right)\right] e^{-\kappa\left(\beta_{v}^{2}+\gamma_{m}^{2}\right)} \\
+ & \left.\frac{\sinh \left[\left(\gamma_{m}^{2}-\frac{\omega}{\kappa}\right)^{1 / 2}\left(z+\frac{\ell}{2}\right)\right]}{\sinh \left[\left(\gamma_{m}^{2}-\frac{\omega}{\kappa}\right)^{1 / 2} \ell\right]} e^{-\omega t}\right\}
\end{aligned}
$$

The aforementioned degenerated result agrees with the previous result [3].

\section{Numerical Results, Discussion and Remarks}

For the sake of simplicity of calculation, we introduce the following dimensionless values

$$
\begin{aligned}
& \bar{\xi}=\xi / \xi_{0}, \bar{z}=[z-(-\ell / 2)] / \xi_{0}, e=c / \xi_{0}, \\
& \tau=\kappa t / \xi_{0}^{2}, \bar{\theta}=T / T_{0}, \bar{u}_{i}=u_{i} / \xi_{0} \alpha T_{0}, \\
& \bar{\sigma}_{i j}=\sigma_{i j} / E \alpha T_{0}(i, j=\xi, \eta)
\end{aligned}
$$

Substituting the value of equation (20) in equations of temperature (16), displacements and components of stresses, we obtained the expressions for our numerical discussion. The numerical computations have been carried out for an elliptical plate with physical parameter as $\xi_{0}=$ $1 \mathrm{~m}, \xi_{i}=0.3 \mathrm{~m}, \ell=0.08 \mathrm{~m}$, reference temperature as $150^{\circ} \mathrm{C}$ and $b=0.1,0.3,0.5,0.7$. The thermo-mechanical properties are considered as modulus of elasticity $E=70 \mathrm{GPa}$, Poisson's ratio $v=0.35$, thermal expansion coefficient $\alpha=$ $23 \times 10^{-6} /{ }^{0} \mathrm{C}$, thermal diffusivity $\kappa=84.18 \times 10^{-6} \mathrm{~m}^{2} \mathrm{~s}^{-1}$ and thermal conductivity $\lambda=204.2 \mathrm{Wm}^{-1} \mathrm{~K}^{-1}$. The $q_{2 n, m}=$ $0.0786,0.356,0.8882,1.7691,2.2344,3.7234,4.6731$, $6.5665,7.3673,9.6733,11.2343,14.2722,16.6896$, $19.3825,22.2566,25.2341,28.6731,31.2345,35.0862$, 39.4664 are the positive \& real roots of the transcendental equation $C_{e_{2 n}}\left(\xi_{0}, q\right)=0$. In order to examine the influence of heating on the plate, the numerical calculations were performed for all the variables and numerical calculations are depicted in the following figures with the help of MATHEMATICA software.

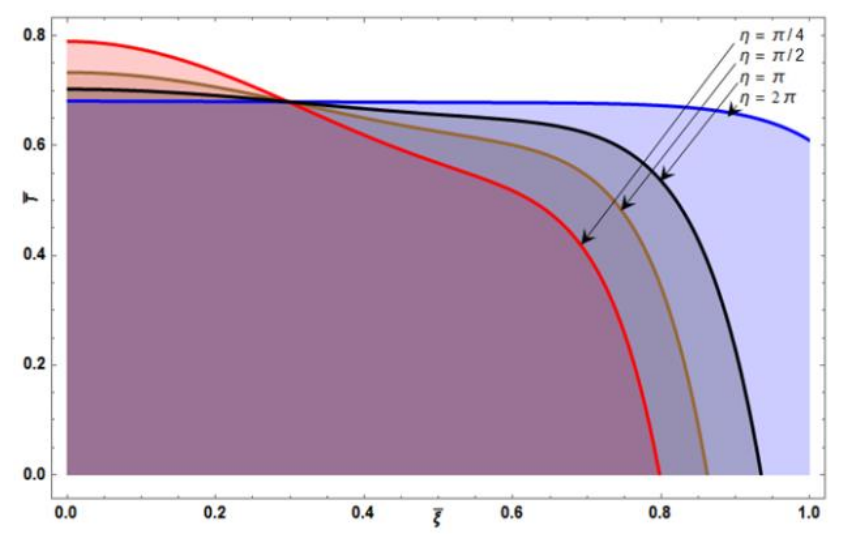

Figure 2 (a): Thermodynamic temperature along $\xi$ direction for various values of $\eta$ and $b=0.4$
Figures. 2-3 illustrates the numerical analysis of two temperature distributions, strains and stresses of the nonsimple elliptical plate due to point impulsive heat sources within the solid, under thermal boundary conditions. Figures 2(a) and 2(b) shows the variation in the thermodynamical and conductive temperature for various angles along the radial direction ( $\xi$-axial direction) for a fixed value of temperature discrepancy $b=0.4$. The maximum temperature can be found at the inner core of the plate due the available point heat source and is lowering at the outer edge of the plate, may be due to thermal insulation of outer edge. Figures 2(a) and 2(b) shows the parameter $b$ has significant effects on temperature field and changes its behaviour as it reaches to the outer boundary along the radial direction.

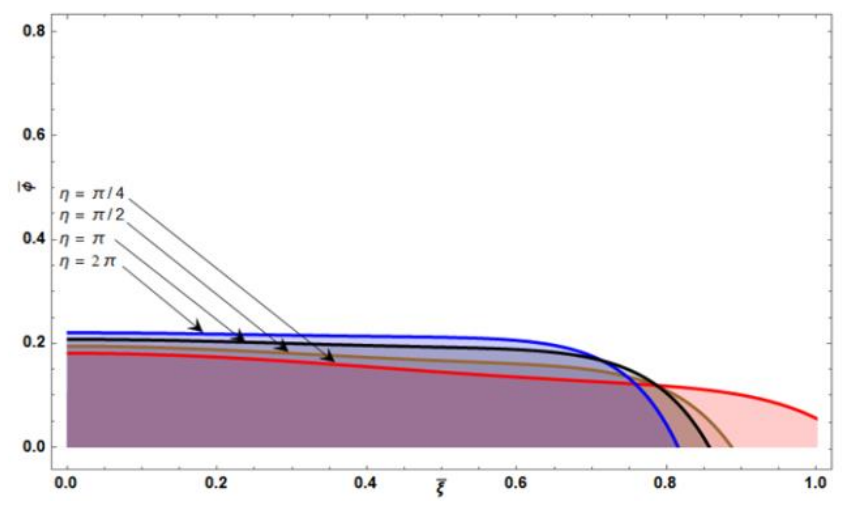

Figure 2 (b): Conductive temperature along $\xi$-direction for various values of $\eta$ and $b=0.4$

Figures 2(c) and 2(d) represents the non-dimensional temperature distribution along the axial direction (i.e. along $z$-axial direction) for the different angles $\eta$. It is observed that the due to the available impulsive heat function at the outer edge, the instantaneous increase in the temperature is found at the outer edge. The linear proportionality of the temperature along the $z$-axis may be due to the thinness of the plate. Again in Figures 2(a) and 2(b) shows the parameter $b$ has significant effects on temperature field along the thickness direction.

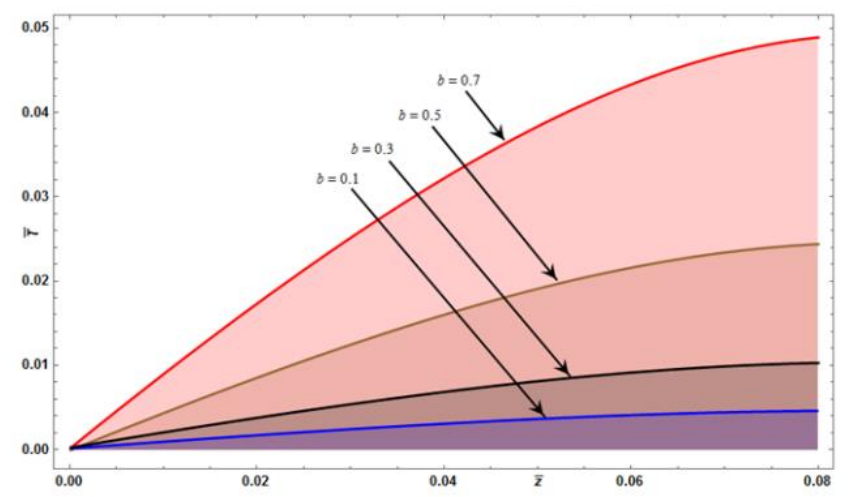

Figure 2 (c): Thermodynamic temperature along zdirection for different values of $b$ and fixed $\eta$

Figures 2(e) and 2(f) express an increase in temperature distribution along the radial direction for the various values of temperature discrepancy $(b=0.1,0.3,0.5,0.7)$. Also, as $b$ increases the thermodynamical $\bar{T}$ and conductive temperature $\bar{\phi}$ decreases in the interval $0<\bar{\xi}<\bar{\xi}_{0}$. The 
temperature field depends not only on the time $t$ and space coordinate $\bar{\xi}$, but also depends on the two-temperature parameter $b$.

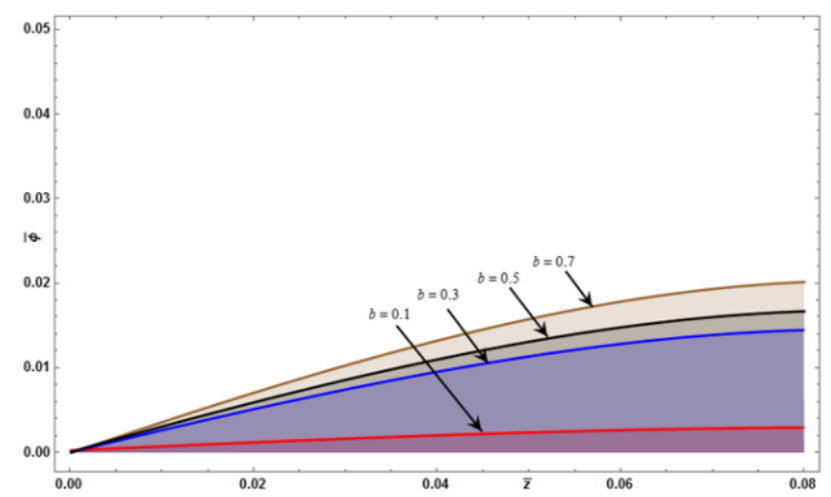

Figure 2 (d): Conductive temperature along z-direction for different values of $b$ and fixed $\eta$

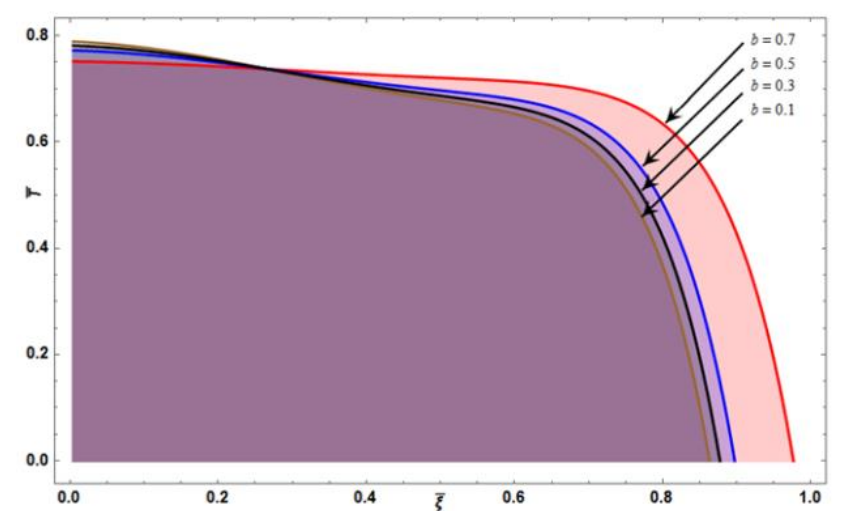

Figure 2 (e): Thermodynamic temperature along $\xi$ direction for different values of $b$ and fixed $\bar{z}$

Figure 3(a) depicts dimensionless thermal stresses along the axial direction. The nature of radial stress and tangential stresses are of the same kind, they attain minimum at the outer face, whereas hoop stress has similar nature as tangential stress but opposite in nature. The contrary nature of the hoop stress can be observed, the stress is increasing from the inner face to the outer face of the plate and these changes could be owing due to thermal expansion.

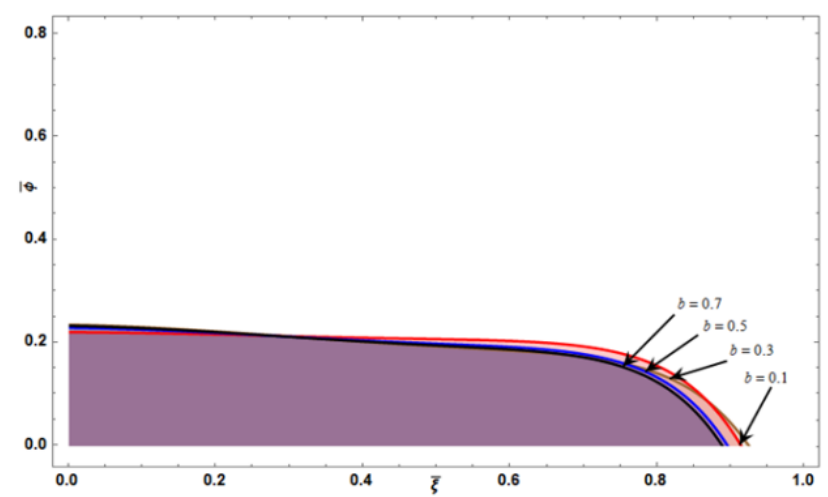

Figure $2(f)$ : Conductive temperature along $\xi$-direction for different values of $b$ and fixed $\bar{z}$

Figure 3(b) illustrates the absolute value of dimensionless stresses along the radial direction. It is observed that the tangential stress and radial stress increases towards the outer core of the plate, but shear stress decrease along the radius, satisfying the traction free boundary conditions.

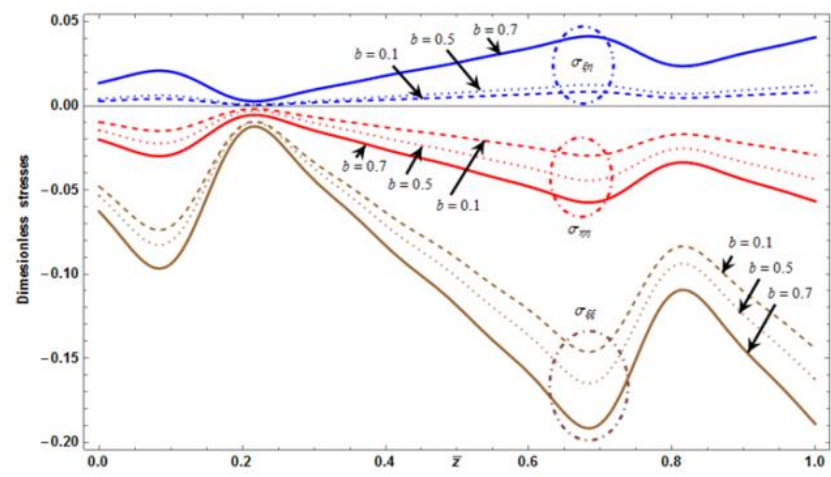

Figure 3 (a): Dimensionless stresses along z-direction for different values of $b$

Figure 3(c) outlines the sinusoidal nature of dimensionless stresses along the angular direction. The tangential stress and radial stress have the same kind of amplitude at the central core of the plate, whereas circumferential shear stress has exactly opposite nature of that tangential stress and radial stress. It can be narrated that the dimensionless stresses portray the periodicity $\eta=n \pi, n=1,2,3 \ldots$.

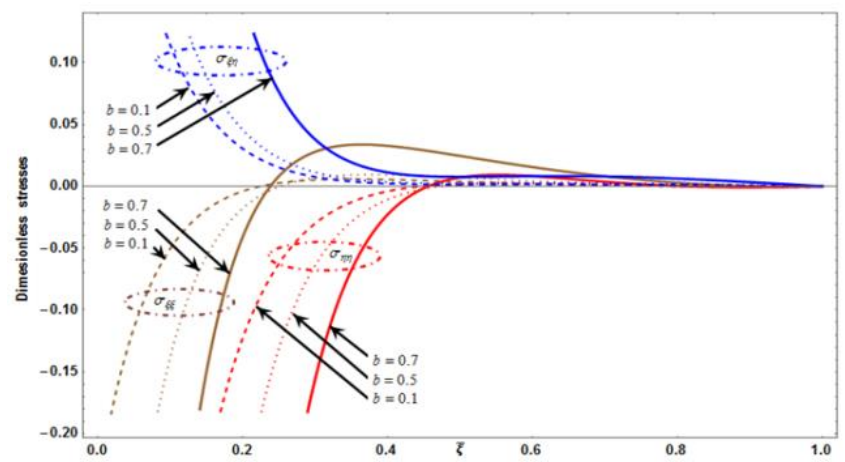

Figure $3(b)$ : Dimensionless stresses along $\xi$-direction for different values of $b$

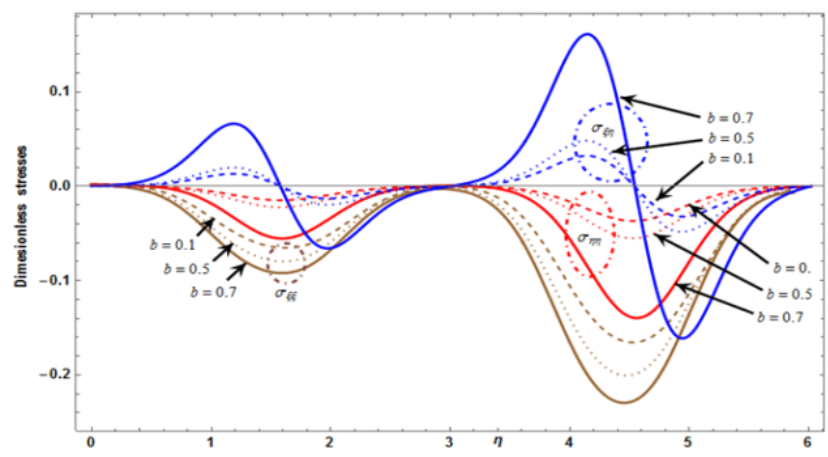

Figure 3 (c): Dimensionless stresses along $\eta$-direction for different values of $b$

In the preliminary investigation, as expected, there is an increment in the rate of heat propagation with a radius, which leads to compressive force in the outer surface and expands more on the inner part. To satisfy the convergence of series and traction-free conditions at an arbitrary point on the boundary, we must replace $\Sigma_{\infty}$ in the stress components by $\Sigma_{20}$, approximately. 


\section{Conclusion}

Motivated by the earlier analysis made up of non-simple elastic material, we have proposed the analytical solution of transient thermal stress problem in a thin elliptical plate made up of non-simple elastic material subjected to point impulsive time-dependent source of heat moving with constant velocity over the specified finite portion. To the author's knowledge, there have been no reports of the solution so far in which sources are generated according to the linear function of the temperature in mediums in the form of an elliptical plate of finite height with Dirichlet type boundary conditions. The analysis of non-stationary threedimensional equation of heat conduction is investigated involving the Mathieu and modified functions by employing the Laplace transform technique when there are conditions of Dirichlet type contour acting on the object under consideration. The following results were obtained to be carried out during our research are:

- The advantage of this method is its generality and its mathematical power to handle different types of mechanical and thermal boundary conditions in both simple and non-simple materials.

o The greatest tensile stress is moving from the focal center to the external area might be because of heat, stress, focus or accessible interior heat sources under considered temperature distribution.

- The outcomes presented in this paper should prove subsidiary for researchers in technical and manufacturing, as well as for those working on the advance of mechanics of solids.

- At last, the most extreme tensile stress happens in the roundabout center on the major axis contrasted with the circular focal part shows the dispersion of weak heating. It may be because of lacking entrance of heat through the circular inward surface.

\section{Acknowledgements}

One of the authors would like to extend the utmost gratitude to Prof. N.W. Khobragade for his counsel, confidence, patience and constructive criticism throughout the research.

\section{References}

[1] P.J. Chen and M.E. Gurtin, "On a theory of heat conduction involving two temperatures," Zeitschrift für Angew Mathematik und Physik, 19, 614-627, 1968.

[2] Chen, M.E. Gurtin and W.O. Willams, "On the thermodynamics of non-simple Elastic material with two temperatures," Zeitschrift für Angew Mathematik und Physik, 20(1), 107-112, 1969.

[3] S.K. Roy Choudhary, "A note on quasi-static thermal deflection of a thin clamped circular plate due to ramptype heating of a concentric circular region of the upper face," J. Franklin Inst.,206, 1973.

[4] N.L. Khobragade and K.C. Deshmukh, "Thermoelastic problem of a thin circular plate subjected to a distributed heat supply," Journal of Thermal Stresses, 28, 171-184, 2005.

[5] V. Varghese and L. Khalsa, "Transient thermoelastic problem for a thick annular disc with radiation," Int. J. of Appl. Math and Mech., 7, 57-73, 2011.
[6] P. Bhad, V. Varghese, L. Khalsa, "Heat source problem of thermoelasticity in an elliptic plate with thermal bending moments," J. of Thermal Stresses, 40, 96-107, 2016.

[7] P. Bhad, V. Varghese, L. Khalsa, “A modified approach for the thermoelastic large deflection in the elliptical plate," Archive of Applied Mechanics, 87, 767-781, 2016.

[8] P. Bhad, V. Varghese, L. Khalsa, "Thermoelasticinduced vibrations on an elliptical disk with internal heat sources," J. of Thermal Stresses, 40, 96-107, 2016.

[9] P. Bhad, V. Varghese, L. Khalsa, "Thermoelastic theories on elliptical profile objects: An overview prospective." Int. J. Adv. Appl. Math Mech., 4, 12-20, 2016.

[10] T. Dhakate, V. Varghese, L. Khalsa, "Integral transform approach for solving dynamic thermal vibrations in the elliptical disk," J. of Thermal Stresses, 40, 1093-1110, 2017.

[11] T. Dhakate, V. Varghese, L. Khalsa, "A Green's function approach for the thermoelastic analysis of an elliptical cylinder," Int. J. Adv. Appl. Math. Mech, 5, 3040, 2017.

[12] T. Dhakate, V. Varghese, L. Khalsa, "An analytical solution for transient asymmetric heat conduction in a multilayer elliptic annulus and its associated thermal stresses," Int. J. Math. Appl., 6, 1093-29-42, 2018.

[13] V. Varghese, T. Dhakate, Lalsingh Khalsa, "Thermoelastic vibrations in a thin elliptic annulus plate with elastic supports," Theor. Appl. Mech. Lett., 8, 32-42, 2018.

[14] S. Chakrabarty, "One-dimensional thermoelastic wave in a non-simple medium," Bull. Calcutta Math. Soc., 64, 129-135, 1972.

[15] R. Laha, "One-dimensional thermal shock problem of thermoelasticity in a non-simple medium," Indian $J$. Pure Appl. Math., 43, 421-431, 1977.

[16] A.K. Dhar, "Heat-source problem of thermoelasticity in a non-simple elastic medium," Indian J. Pure Appl. Math., 13, 1384-1392, 1982.

[17] A.K. Dhar, "Mechanical shock problem of coupled thermoelasticity in a non-simple elastic material," Indian J. Pure Appl. Math., 16, 174-178, 1985.

[18] M. Ciarletta, "Thermoelasticity of non-simple materials with thermal relaxation," J. Thermal Stresses, 19(8), 731748, 1996.

[19] H.S. Chakraborty, A.K. Sengupta, K. Ghosh, "On a problem of coupled thermoelasticity in a non-simple medium," Analele S. Ale Universit at, II "AL.I.CUZA"

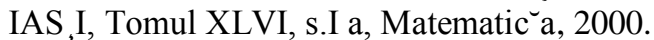

[20] R. Quintanilla, "Thermoelasticity without energy dissipation of non-simple materials, ZAMM" J. Appl. Math. Mech., 83, 172-180, 2003.

[21] R. Quintanilla, “On existence, structural stability, convergence and spatial behaviour in thermoelastic with two temperature," Acta Mech., 168, 161-173, 2004. 
[22] H.D.F Sare, J.E.M. Rivera, R. Quintanilla, "Decay of solutions in non-simple thermoelastic bars," Int. J. Eng. Sci., 48, 1233-1241, 2010.

[23] A.M. Zenkour and A.E. Abouelregal, "Non-simple magneto-thermoelastic solid cylinder with variable thermal conductivity due to harmonically varying heat," Earthquakes Structures, 10, 681-697, 2016.
[24] S. Ghosh, "On the solution of the equations of elastic equilibrium suitable for elliptic boundaries," Trans. Am. Math. Soc., 32, 47-60, 1930.

[25] Y. Sugano, Y. Kondoh and H. Yano, “An analytical for a plane thermal stress problem expressed in elliptical coordinates," JSME, 56, 78-83, 1990.

[26] N.W. McLachlan, Theory and Application of Mathieu function, Oxford Univ. Press, 1947. 